\title{
Exploring a two-criterion order scheduling problem by using five heuristics
}

\author{
Win-Chin Lin $^{a}$, Carol Yu ${ }^{b}$, Shuenn-Ren Cheng ${ }^{c}$, Tzu-Yun Lin $^{a}$, \\ Yuan-Po Chao $^{d}$, Shang-Chia Liu ${ }^{e}$, and Chin-Chia Wu ${ }^{a^{*}}$ \\ a Department of Statistics, Feng Chia University,Taichung, 40724, Taiwan \\ ${ }^{\mathrm{b}}$ Fundraising office, Fu Jen Catholic University Hospital, New Taipei City, 24205, Taiwan \\ ${ }^{\mathrm{c}}$ Graduate Institute of Business Administration, Cheng Shiu University, Kaohsiung, Taiwan \\ ${ }^{\mathrm{d}}$ Department of Business Administration, Cheng Shiu University, Kaohsiung, Taiwan \\ ${ }^{\mathrm{e}}$ Department of Business Administration, Fu Jen Catholic University,New Taipei City,Taiwan \\ ${ }^{*}$ Corresponding author: Chin-Chia Wu, cchwu@fcu.edu.tw
}

\begin{abstract}
Recently the order scheduling (OS) problem is concerned by the research community. However, the OS study with more than one criterion is only few. In view of this limitation, we address an OS problem in which the objective is to find a schedule to minimize the sum of total flowtime and the maximum tardiness. The complexity of this problem is very difficult. Thus, we use five heuristics including three modified heuristics, an iterated greedy (IG) method, and a particle swarm colony (PSO) algorithm for finding approximately solutions. Finally, the statistical results and comparison performances of all five heuristics are reported.
\end{abstract}

Key words: order scheduling; particle swarm optimization; iterated greedy; total flowtime; maximum tardiness

\section{Introduction}

In the recent days, the issue of customer order scheduling has grown a hot toptic of research. Some applications of the OS model have existed in the production of integrated circuits and in the manufacturing of semifinished lenses (see Ahmadi et al. ${ }^{1}$ ).

The OS literature on minimizing the total completion time criterion, readers can refer to Leung et al. ${ }^{2,3}$, Wagneur and Sriskandarajah ${ }^{4}$, Wang and Cheng ${ }^{5}$, and Sung and Yoon ${ }^{6}$; The OS works on minimizing the total weighted order completion time, readers may refer to focus on discussing the complexity of the problem on two machines by Sung and Yoon $^{6}$ and Ahmadi and Bagchi ${ }^{7}$, and to on analysing some approximation algorithms and worst bounds by Leung et al. ${ }^{8-11}$, Wang and Cheng ${ }^{5}$, and Chen and Hall ${ }^{12}$, etc. As the OS literature involving due dates, we refer readers to Blocher et al. ${ }^{13}$, Erel and Ghosh ${ }^{14}$, Hsu and Liu ${ }^{15}$, Lee $^{16}$ used a branch-and-bound and several heuristics to solve a mean tardiness OS problem. Leung et al. ${ }^{17}$, Yang ${ }^{18}$, and Xu et al. ${ }^{19}$ studied a OS model with a learning effect to minimize the total tardiness. They develop a branch-and-bound algorithm, simulated annealing, particle swarm optimization, and order-scheduling MDD algorithms for it. More recently, Framinan and Perez-Gonzalez ${ }^{20}$ propose a greedy search algorithm for the customer order scheduling problem to minimize the total completion time. They propose a greedy search algorithm and compared the proposals with existing approximate algorithms. Lin et al. ${ }^{21}$ considered a twoagent multi-facility order scheduling with ready times. The objective is to minimize the total 
completion time of the orders of one agent, with the restriction that the total completion time of the orders of the other agent cannot exceed a given limit.

In light of the above OS literature focuses on single criterion, however, there are common encountered more than two criteria in many real situations. This motivates us to explore minimizing the sum of the total flowtime and maximum tardiness as the objective function. Minimizing the total completion time means that the system can yield an efficient task planning to reduce carrying costs, while, minimizing the maximum tardiness of all jobs means that managers reduce the penalty costs from outside customers. To be best our knowledge, this problem has not been explored until now. The remainder of this study is organized as follows: In Section 2, we define the proposed model. In Section 3, we introduce the details of IG and PSO algorithms and several heuristics. In Section 4, we provide observations of all the proposed algorithms. We draw the conclusions and offer suggestions in the last section.

\section{Problem statement}

The study can be formally described as follows. Consider $n$ orders which are operated on $m$ different machines. Those machines are designed in parallel. Pre-emption and machine breakdown are not allowed. Let $t_{i k}$ be the processing time on machine $M_{k}$ and let $d_{i}$ be the due date for order $i$. All the $n$ orders are ready at time zero. The objective function of this study is to find a schedule to minimize the sum of the total flowtime and maximum tardiness, i.e., minimize $\sum C_{i}(S)+T_{\max }(S)$. This problem is also NP-hard because the total flowtime minimization OS problem has been shown as NP-hard by Ahmadi et al. ${ }^{1}$. Therefore, we propose some heuristics, an IG algorithm, and a PSO for near-optimal solutions.

\section{Methods}

Due to the fact that this problem is not easily to find the optimal solution in a short time, we then propose three heuristics which are modified from Smith ${ }^{20}$ and Van Wassenhove and Golders $^{22}$. The main idea of three heuristics is that we replace the processing time by the maximum value, the mean value, and the minimum value among m machines, respectively. Then we apply Van Wassenhove and Golders's algorithm to find all possible feasible solutions, at last, we choose the one with the minimum objective solution among all the feasible solutions. They are recorded as VW-H1, VW-H2, and VW-H3. For more details of Van Wassenhove and Golders's algorithm, readers may refer to Van Wassenhove and Golders ${ }^{22}$. In what follows, we then propose an IG and a PSO to find near-optimal solutions. For the details of IG method, readers may refer to Ruiz and Stützle ${ }^{24}$, while for the details of PSO, readers may refer to Shi and Eberhart ${ }^{25}$ and Eberhart and Kennedy ${ }^{26}$. According our preliminary instances, the parameters adopted in the IG algorithm, namely the controlling temperature parameter $(T)$, the number of neighborhood improvements $(M)$, and the number of destructions $(d)$, are set to $\mathrm{T}=0.75, \mathrm{M}=600$, and $\mathrm{d}=\mathrm{n} / 2$. Meanwhile, based on the experimental pretests of Xu et al. ${ }^{19}$, the parameters (w, B1, B2, N, NN, ITRN) $=(0.5,0.5,0.5$, $\mathrm{N}, \mathrm{NN} / 2,600$ ) were used in the PSO method.

\section{The tested results}

In this section we test and report a computational experiment to evaluate the performances of 
all proposed five methods over the small and big numbers of orders, respectively. The processing times of orders are generated from a uniform distribution $U(1,100)$ based on the designed in Leung et al. ${ }^{17}$, Lee ${ }^{16}$ and Xu et al. ${ }^{19}$, while the due dates of orders are generated from another uniform distribution $T P T \times U(1-\tau-R / 2,1-\tau+R / 2)$, where $T P T=\sum_{i=1}^{m} \sum_{j=1}^{n} t_{i j} / m, \tau$ is noted the tardiness, and $\mathrm{R}$ is noted the range of the due dates. The values of $(\tau, R)$ are set at $(0.25,0.25),(0.5,0.25),(0.5,0.5),(0.5,0.75),(0.25,0.5)$, and $(0.25,0.75)$. One hundred instances are tested for each case.

In what follows, we will evaluate the performance levels of the branch-and-bound and five heuristics for small number of orders. We examine two order sizes at $n=12$ and 14 and three machine sizes at $m=2,3$, and 4 . One hundred instances were tested for each case. A total of 3600 instances were tested for small numbers of orders. For each heuristic method, we report the average gap (AGP), where, $V i$ is the objective function yielded by each heuristic, and $V^{*}$ is the smallest value of the objective function among the five proposed algorithms. The results are summarized in Tables 1-3.

As shown in Tables 1 and 3, the AGPs of five heuristics increase as the value of $m$ or $R$ increases. On the other hand, Table 2 reports that the AGPs of five heuristics decrease as the value of $\tau$ increases.

Table 1 -The gap performance of five methods when $m$ changes $(n=12,14)$

\begin{tabular}{cccrcrr}
\hline $\mathrm{n}$ & $\mathrm{m}$ & IG & PSO & VW-H1 & VW-H2 & VW-H3 \\
\hline 12 & 2 & 430.158 & 433.063 & 448.380 & 445.497 & 482.285 \\
& 3 & 480.050 & 483.260 & 510.327 & 503.060 & 552.025 \\
& 4 & 515.533 & 518.222 & 551.198 & 543.567 & 595.918 \\
14 & 2 & 501.358 & 506.058 & 522.447 & 518.072 & 560.253 \\
& 3 & 550.527 & 554.758 & 581.258 & 575.482 & 630.257 \\
& 4 & 615.090 & 619.518 & 654.092 & 641.600 & 718.783 \\
\hline
\end{tabular}

Table 2 -The gap performance of five methods when $\tau$ changes $(\mathrm{n}=12,14)$

\begin{tabular}{cccrcrr}
\hline $\mathrm{N}$ & $\tau$ & IG & PSO & VW-H1 & VW-H2 & VW-H3 \\
\hline 12 & 0.25 & 519.856 & 523.209 & 551.297 & 544.838 & 598.812 \\
& 0.50 & 430.639 & 433.154 & 455.307 & 449.911 & 488.007 \\
14 & 0.25 & 608.300 & 613.099 & 641.024 & 632.486 & 701.367 \\
& 0.50 & 503.017 & 507.124 & 530.840 & 524.283 & 571.496 \\
\hline
\end{tabular}

Table 3 -The gap performance of five methods when $\mathrm{R}$ changes $(\mathrm{n}=12,14)$

\begin{tabular}{ccccccc}
\hline $\mathrm{n}$ & $\mathrm{R}$ & $\mathrm{IG}$ & $\mathrm{PSO}$ & $\mathrm{VW}-\mathrm{H} 1$ & $\mathrm{VW}-\mathrm{H} 2$ & $\mathrm{VW}-\mathrm{H} 3$ \\
\hline 12 & 0.25 & 432.817 & 435.345 & 460.325 & 455.560 & 494.698 \\
& 0.50 & 481.127 & 483.773 & 510.223 & 502.233 & 550.662 \\
& 0.75 & 511.798 & 515.427 & 539.357 & 534.330 & 584.868 \\
14 & 0.25 & 496.822 & 499.990 & 524.252 & 519.253 & 567.717 \\
& 0.50 & 555.977 & 560.245 & 587.543 & 578.235 & 634.287 \\
& 0.75 & 614.177 & 620.100 & 646.002 & 637.665 & 707.290 \\
\hline
\end{tabular}

Next, we examine the performances of all five proposed algorithms for large numbers of orders. We set the order sizes at $n=50$ and 100 and the machine sizes at $m=5,10$, and 20 . We design the values of $(\tau, R)$ at $(0.25,0.25),(0.5,0.25),(0.5,0.5),(0.5,0.75),(0.25,0.5)$, 
and $(0.25,0.75)$. One hundred instances are examined for each case. Therefore, in total, 3600 instances are tested. The related results are summarized in Tables 4-6.

Tables 4 and 6 release that the AGPs of five heuristics increase as the value of $m$ or $R$ increases. Meanwhile, the AGPs of five heuristics decrease as the value of ${ }_{\tau}$ increases from Table 5.

Table 4 -The gap performance of five methods when $m$ changes $(n=50,100)$

\begin{tabular}{ccccccc}
\hline $\mathrm{n}$ & $\mathrm{m}$ & $\mathrm{IG}$ & $\mathrm{PSO}$ & $\mathrm{VW}-\mathrm{H} 1$ & $\mathrm{VW}-\mathrm{H} 2$ & $\mathrm{VW}-\mathrm{H} 3$ \\
\hline 50 & 5 & 2095.873 & 2191.715 & 2227.650 & 2145.530 & 2302.747 \\
& 10 & 2247.800 & 2328.432 & 2426.975 & 2332.222 & 2484.847 \\
& 20 & 2374.215 & 2443.653 & 2564.323 & 2483.867 & 2614.037 \\
100 & 5 & 4149.487 & 4407.415 & 4342.553 & 4151.592 & 4441.107 \\
& 10 & 4391.645 & 4590.623 & 4675.648 & 4468.648 & 4740.100 \\
& 20 & 4579.588 & 4747.620 & 4890.538 & 4704.155 & 4939.408 \\
\hline
\end{tabular}

Table 5 -The gap performance of five methods when $\tau$ changes $(\mathrm{n}=50,100)$

\begin{tabular}{ccccccc}
\hline $\mathrm{n}$ & $\tau$ & IG & PSO & VW-H1 & VW-H2 & VW-H3 \\
\hline 50 & 0.25 & 2440.314 & 2530.111 & 2622.483 & 2528.909 & 2689.804 \\
& 0.50 & 2038.278 & 2112.422 & 2190.149 & 2112.170 & 2244.616 \\
100 & 0.25 & 4766.616 & 4993.281 & 5053.203 & 4839.690 & 5129.959 \\
& 0.50 & 3980.531 & 4170.491 & 4219.290 & 4043.240 & 4283.784 \\
\hline
\end{tabular}

Table 6 -The gap performance of five methods when $\mathrm{R}$ changes $(\mathrm{n}=50,100)$

\begin{tabular}{ccccccc}
\hline $\mathrm{n}$ & $\mathrm{R}$ & $\mathrm{IG}$ & $\mathrm{PSO}$ & $\mathrm{VW}-\mathrm{H} 1$ & $\mathrm{VW}-\mathrm{H} 2$ & $\mathrm{VW}-\mathrm{H} 3$ \\
\hline 50 & 0.25 & 2039.253 & 2111.595 & 2193.762 & 2111.738 & 2243.737 \\
& 0.50 & 2275.077 & 2359.678 & 2442.798 & 2357.447 & 2508.227 \\
& 0.75 & 2403.558 & 2492.527 & 2582.388 & 2492.433 & 2649.667 \\
100 & 0.25 & 3988.890 & 4176.543 & 4224.778 & 4050.163 & 4289.105 \\
& 0.50 & 4447.842 & 4660.033 & 4714.237 & 4516.082 & 4786.802 \\
& 0.75 & 4683.988 & 4909.082 & 4969.725 & 4758.150 & 5044.708 \\
\hline
\end{tabular}

Finally, we investigate the statistical differences in the performance levels of the five algorithms. We apply one-way analysis of variance for small and large orders. The results are provided in Tables 7 and 8 . In view of the fact that the $p$-values are less than 0.05 , one can refer to the row "Algorithm" under "Source of Variation" in Tables 7 and 8, to confirm that the performance differences of the proposed five algorithms are significant at the 0.05 level, regardless of the order size.

Table 7 -The ANOVA of five methods $(\mathrm{n}=12,14)$

\begin{tabular}{crrrrr}
\hline Source of Variation & DF & Sum of Squares & Mean Square & F Value & Pr $>$ F \\
\hline Model & 10 & 1626761.48 & 162676.14 & 346.49 & $<.0001$ \\
Algorithm & 4 & 127635.46 & 31908.86 & 67.96 & $<.0001$ \\
Size & 1 & 316014.82 & 316014.82 & 673.10 & $<.0001$ \\
$\tau$ & 1 & 487196.43 & 487196.43 & 1037.71 & $<.0001$ \\
$\mathrm{R}$ & 2 & 315541.99 & 157770.99 & 336.05 & $<.0001$ \\
Machine & 2 & 380372.75 & 190186.37 & 405.09 & $<.0001$ \\
Error & 169 & 79344.02 & 469.49 & & \\
Corrected Total & 179 & 1706105.51 & & & \\
\hline
\end{tabular}


Table 8 -The ANOVA of five methods $(\mathrm{n}=50,100)$

\begin{tabular}{crrrrrr}
\hline Source of Variation & DF & Sum of Squares & Mean Square & F Value & Pr $>$ F \\
\hline Model & 10 & 250411836.5 & 25041183.6 & 1030.97 & $<.0001$ \\
Algorithm & 4 & 1772423.1 & 443105.8 & 18.24 & $<.0001$ \\
Size & 1 & 217222794.9 & 217222784.9 & 8943.24 & $<.0001$ \\
$\tau$ & 1 & 17294652.2 & 17294652.2 & 712.03 & $<.0001$ \\
& $\mathrm{R}$ & 2 & 9559010.9 & 4779505.4 & 196.78 & $<.0001$ \\
Machine & 2 & 2562955.4 & 2281477.7 & 93.93 & $<.0001$ \\
Error & 169 & 4104850.7 & 24289.1 & & \\
Corrected Total & 179 & 254516687.2 & & & \\
\hline
\end{tabular}

To further compare the quality of solutions among the five algorithms, SAS (version 9.4) was used to execute Fisher's least significant difference tests. The results are provided in Tables 9 and 10. As shown in Table 9, the overall averages (for small orders) of AGPs, in decreasing order, are 589.920, 544.617, 537.879, 519.147, and 515.453 for VW-H3, VW-H1, VW-H2, PSO, and IG, respectively. All pairwise comparisons between the five algorithms are significant at the 0.05 level for small orders, except those of the IG vs. PSO and VW-H1 vs. VW-H2. As shown in Table 10, the overall averages (for big orders) of AGPs, in decreasing order, are 3587.04, 3521.28, 3451.58, 3381.00, and 3306.43 for VW-H3, VW-H1, PSO, VW$\mathrm{H} 2$, and IG, respectively. All pairwise comparisons between the five algorithms are significant at the 0.05 level for large orders, except those of the PSO vs. VW-H1, PSO vs. VW-H1 and VW-H1vs. VW-H3.

Table 9 -The Fisher' s LSD of five methods $(\mathrm{n}=12,14)$

\begin{tabular}{lcc}
\hline $\begin{array}{c}\text { Pairwise Comparison } \\
\text { Between Algorithms }\end{array}$ & $\begin{array}{c}\text { Pairwise Mean Difference } \\
\mid \overline{G A P}-\overline{G A P_{J} \mid}\end{array}$ & $\begin{array}{c}\text { LSD }(\alpha=0.05)=10.082 \\
\text { Difference }>\text { LSD? }\end{array}$ \\
\hline IG vs. PSO & $|515.453-519.147|$ & No \\
IG vs. VW-H1 & $|515.453-544.617|$ & Yes \\
IG vs. VW-H2 & $|515.453-537.879|$ & Yes \\
IG vs. VW-H3 & $|515.453-589.920|$ & Yes \\
PSO vs. VW-H1 & $|519.147-544.617|$ & Yes \\
PSO vs. VW-H2 & $|519.147-537.879|$ & Yes \\
PSO vs. VW-H3 & $|519.147-589.920|$ & Yes \\
VW-H1 vs. VW-H2 & $|544.617-537.879|$ & No \\
VW-H1 vs. VW-H3 & $|544.617-589.920|$ & Yes \\
VW-H2 vs. VW-H3 & $|537.879-589.920|$ & Yes \\
\hline
\end{tabular}

These results report that the mean AGP for small orders or for large orders of the IG algorithm is the smallest (best), whereas VW-H3 is the largest (worst) for both of small and big orders, at the 0.05 significance level. Additionally, the IG has the least dispersion of the five algorithms, as shown in Figs 1 and 2. These results show that the solutions obtained from the proposed IG have both high accuracy and high stability. 
Table 10 -The Fisher' s LSD of five methods $(\mathrm{n}=50,100)$

\begin{tabular}{lcc}
\hline $\begin{array}{r}\text { Pairwise Comparison } \\
\text { Between Algorithms }\end{array}$ & $\begin{array}{c}\text { Pairwise Mean Difference } \\
\mid \overline{G A P}-\overline{G A P_{J} \mid}\end{array}$ & $\begin{array}{c}\operatorname{LSD}(\alpha=0.05)=72.517 \\
\text { Difference }>\text { LSD? }\end{array}$ \\
\hline IG vs. PSO & $|3306.43-3451.58|$ & Yes \\
IG vs. VW-H1 & $|3306.43-3521.28|$ & Yes \\
IG vs. VW-H2 & $|3306.43-3381.00|$ & Yes \\
IG vs. VW-H3 & $|3306.43-3587.04|$ & Yes \\
PSO vs. VW-H1 & $|3451.58-3521.28|$ & No \\
PSO vs. VW-H2 & $|3451.58-3381.00|$ & No \\
PSO vs. VW-H3 & $|3451.58-3587.04|$ & Yes \\
VW-H1 vs. VW-H2 & $|3521.28-3381.00|$ & Yes \\
VW-H1 vs. VW-H3 & $|3521.28-3587.04|$ & No \\
VW-H2 vs. VW-H3 & $|3381.00-3587.04|$ & Yes \\
\hline
\end{tabular}

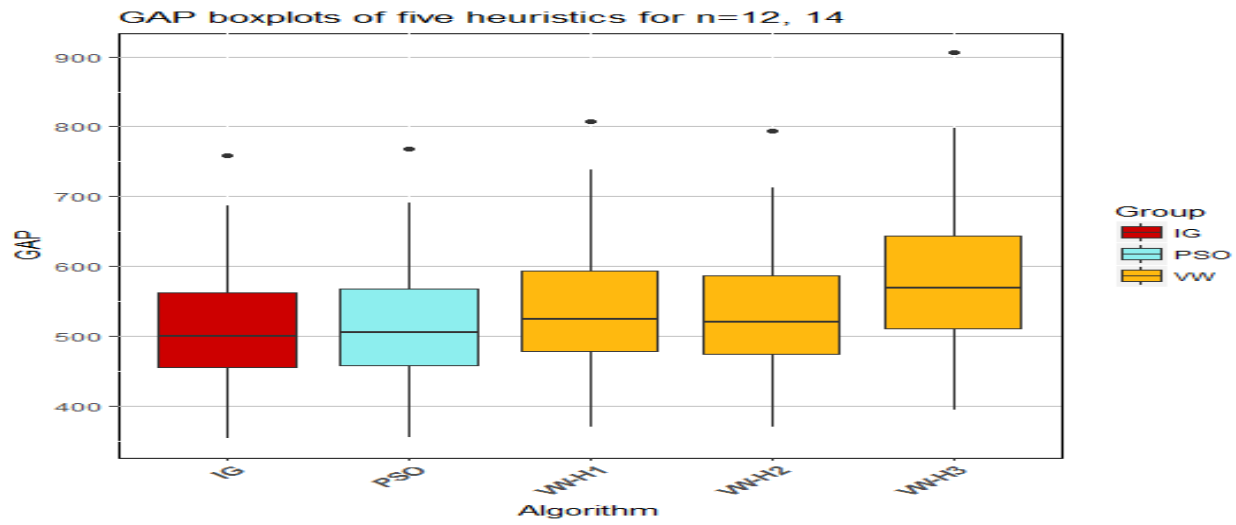

Fig. 1 -The boxplots of five methods $(\mathrm{n}=12,14)$

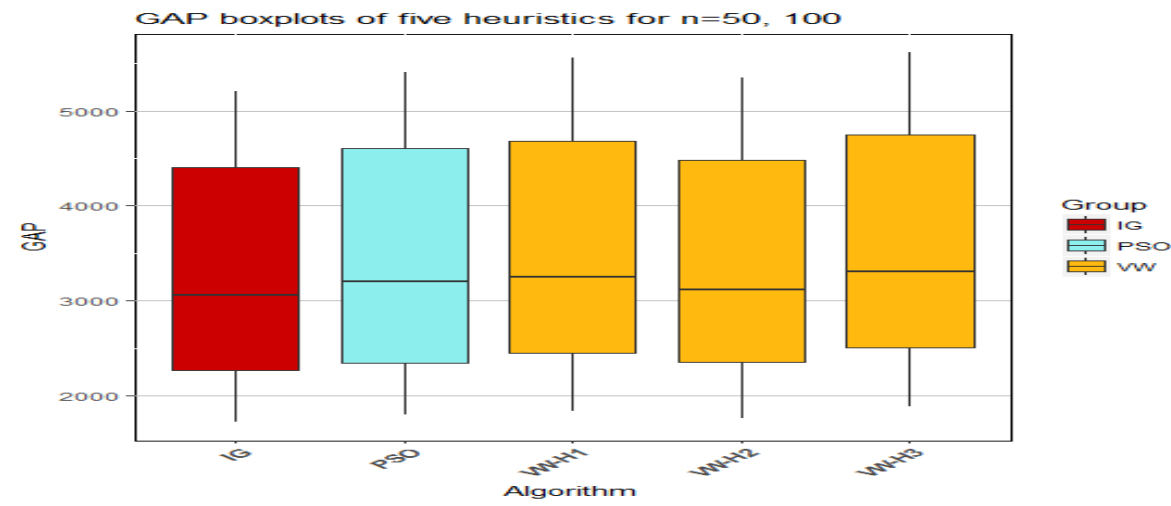

Fig. 2-The boxplots of five methods $(\mathrm{n}=50,100)$

\section{Conclusions and suggestions}

The literature releases that OS problems exist in numerous manufacturing and service environments. Production managers commonly should consider optimizing multiple objectives instead of a single objective in many real-life situations. Thus, we address an order scheduling model to minimize the sum of the total flowtime and the maximum tardiness. Different from existed papers assuming that the orders are paid attention on single criterion, this paper addresses the bi-criteria objective function. We then propose five heuristics such as IG, PSO, VW-H1, VW-H2, and VW-H3 methods to find near-optimal solutions. It is noted that IG and PSO are two metaheuristics instead of heuristics. Sometimes, they are not easily 
to construct. The test results confirm that IG can find a good quality of solutions in terms of their high accuracy and stability for both small and large numbers of orders.

\section{Acknowledgement}

This paper was supported by the Ministry of Science Technology (MOST) of Taiwan under grant numbers MOST 105-2221-E-035-053-MY3.

\section{References}

1. R.H. Ahmadi, U. Bagchi, T.A. Roemer, Coordinated scheduling of customer orders for quick response, Naval Research Logistics 52 (2005) 493-512.

2. J.Y.T. Leung, H. Li, M. Pinedo, Order scheduling in an environment with dedicated resources in parallel. Journal of Scheduling 8 (2005) 355-386.

3. J.Y.T. Leung, H. Li, M. Pinedo, Approximation algorithms for minimizing total weighted completion time of orders on identical machines in parallel. Naval Research Logistics 53 (2006a) 243-260.

4. E. Wagneur, C. Sriskandarajah, Open shops with jobs overlap, European Journal of Operational Research 71 (1993) 366-378.

5. G. Wang, , T.C.E. Cheng, Customer order scheduling to minimize total weighted completion time, Proceedings of the 1st Multidisciplinary Conference on Scheduling Theory and Applications (2003) 409-416.

6. C.S. Sung, S.H. Yoon, Minimizing total weighted completion time at a pre- assembly stage composed of two feeding machines. International Journal of Production Economics 54 (1998) 247-255.

7. R.H. Ahmadi, U, Bagchi, Coordinated scheduling of customer orders, Working paper, John E. Anderson Graduate School of Management, University of California, Los Angeles, 1993.

8. J.Y.T. Leung, H. Li, M. Pinedo, Scheduling orders for multiple product types to minimize total weighted completion time, Discrete Applied Mathematics 155 (2007a) 945-970.

9. J.Y.T. Leung, H. Li, M. Pinedo, J. Zhang, Minimizing total weighted completion time when scheduling orders in a flexible environment with uniform machines. Information Processing Letters 103 (2007b) 119-129.

10. J.Y.T. Leung, C.Y. Lee, C.W. Ng, G.H. Young, Preemptive multiprocessor order scheduling to minimize total weighted flowtime. European Journal of Operational Research 190 (2008a) 40-51.

11. J.Y.T. Leung, H. Li, M. Pinedo, Scheduling orders on either dedicated or flexible machines in parallel to minimize total weighted completion time. Annals of Operations Research 159 (2008b) 107-123.

12. Z.L. Chen, N.G. Hall, Supply chain scheduling: assembly systems, Working Paper, Department of Systems Engineering, University of Pennsylvania (2001).

13. J.D. Blocher, D, Chhajed, M, Leung, Customer order scheduling in a general job shop environment, Decision Sciences 29(4) (1998) 951-981. 
14. E. Erel, J.B. Ghosh, Customer order scheduling on a single machine with family setup times: Complexity and algorithms, Applied Mathematics and Computation 185 (2007) 11-18.

15. S.Y. Hsu, C.H. Liu, Improving the delivery efficiency of the customer order scheduling problem in a job shop, Computers \& Industrial Engineering 57 (2009) 856-866.

16. I.S. Lee, Minimizing total tardiness for the order scheduling problem, International Journal of Production Economics 144 (2013) 128-134.

17. J.Y.T. Leung, H. Li, M. Pinedo, Scheduling orders for multiple product types with due date related objectives. European Journal of Operational Research 168 (2006b) 370389.

18. J. Yang, The complexity of customer order scheduling problems on parallel machines, Computers \& Operations Research 32 (2005) 1921-1939.

19. J. Xu, C.-C. Wu, , Y. Yin, C.L. Zhao, Y.T. Chiou, W.C. Lin, An order scheduling problem with position-based learning effect, Computers \& Operations Research 74 (2016) 175-186.

20. J.M. Framinan, P. Perez-Gonzalez, New approximate algorithms for the customer order scheduling problem with total completion time objective. Computers \& Operations Research 78 (2017) 181-192.

21. W.C. Lin, Y. Yin, S.R. Cheng, T.C.E. Cheng, C.H. Wu, C.-C. Wu, Particle swarm optimization and opposite-based particle swarm optimization for two-agent multifacility customer order scheduling with ready times, Applied Soft Computing, 52 (2017) 877-884.

22. W.E. Smith, Various optimizers for single state production. Naval Research Logistic Quarterly 3 (1956) 59-66.

23. L.N. Van Wassenhove, F. Gelders, Solving a bicriterion scheduling problem. European Journal of Operational Research 4 (1980) 42-48.

24. R. Ruiz, T. Stützle, An Iterated Greedy heuristic for the sequence dependent setup times flowshop problem with makespan and weighted tardiness objectives. European Journal of Operational Research 187 (3) (2008) 1143-1159.

25. Y. Shi, R.C. Eberhart, A modified particle swarm optimizer. Proceedings of IEEE International Conference on Evolutionary Computation (1998) 69-73.

26. R.C. Eberhart, J. Kennedy, A new optimizer using particle swarm theory. Proceedings of the Sixth International Symposium on Micro Machine and Human Science, Nagoya, Japan, (1995) 39-43. Piscataway, NJ: IEEE Service Center. 